\title{
Design and development of a real-time readout electronics system to retrieve data from a square multi-anode photomultiplier tube for neutron gamma pulse shape discrimination
}

\author{
Michal Cieslak, Student Member, IEEE, Kelum Gamage, Member, IEEE,
}

\begin{abstract}
Pulse Shape Discrimination (PSD) algorithms can reliably separate neutrons and gamma-ray photons interacting in a scintillation detector. When implemented in the digital domain, the PSD algorithms allow real-time discrimination between neutron and gamma sources. This paper presents a design of a readout electronics system to retrieve data from a multi-anode photomultiplier tube (MAPMT) for a scintillator based codedaperture neutron imager. The scintillator is to be coupled with Hamamatsu H9500, a square MAPMT, where each anode of the MAPMT is linked to a resistor network to infer the position of incidence of radiation within the scintillant. Additionally, the resistor network output signals are to be filtered through a novel noise reduction circuit to preserve the data corresponding to each pulse. Localised pulses are digitised using high sampling rate Analogue to Digital Converter (ADC). Sampled signals are temporarily stored in a local ping-pong buffer, before being processed on a field programmable gate array (FPGA). Initial results suggest that 150 MSPS rate provides sufficient information for neutron gamma source discrimination using PSD. Parallel real-time signal processing, implemented on the FPGA, enables multi-channel functioning to generate an array of interactions within the scintillator in terms of gamma rays and neutrons.
\end{abstract}

Index Terms-Pulse shape discrimination, Readout electronics, Coded-aperture, Neutron detection, Analogue to digital conversion.

\section{INTRODUCTION}

S CINTILLATOR based radiation detectors have been successfully employed in application fields, such as nuclear medicine [1], [2], homeland security [3] and nuclear decommissioning [4], [5]. In each case, accurate localisation of the radioisotopes is of vital importance, since failing to do so would pose a threat to human lives. Although specific requirements may differ between implementations, each of the above application fields necessitates high signal-to-noise ratio (SNR), as well as spatial resolution of the results.

In a typical single anode scintillation counter the scintillator is optically coupled to a photomultiplier tube (PMT). The photocathode of the PMT transforms photons emitted by the scintillator to photoelectrons. The photoelectrons can then be counted via electronic systems connected to the anode of the PMT. These systems do not account for the position of interaction within the scintillator [6].

M. Cieslak and K. Gamage are with the Engineering Department, Lancaster University, Lancaster, LA1 4YW, UK, e-mail: (m.cieslak@lancaster.ac.uk and k.gamage@lancaster.ac.uk).

978-1-5090-2014-0/16\$31.00 ㄷ 2016 IEEE
Recent research into anisotropic properties of crystalline scintillation materials suggest that some positional information can be inferred from the scintillator response [7]. The localisation of a particle interaction with the scintillant can also be achieved by replacing the PMT with a multi-anode photomultiplier tube (MAPMT).

Flat panel MAPMTs from Hamamatsu Photonics, such as H8500 [8] and H9500 [9], have replaced old type of crosswire MAPMTs, substantially increasing the sensitive area of the similar size device. If coupled to a correct scintillator, they have shown superior spatial resolution performance, when used in gamma cameras [1], [2], [10], [11] and neutron detectors [12]. However, due to a high number of anodes, readout electronics circuits are required to provide sufficient filtering of the extracted signal, as well as cross-talk avoidance.

In this paper a design of a new readout electronics circuit for H9500 MAPMT aimed at neutron/gamma pulse shape discrimination (PSD) is discussed. The event localisation is based on resistive network approach [13] requiring a novel signal filtering implementation to preserve the data corresponding to each interaction within the scintillator. The $\mathrm{X}$ and $\mathrm{Y}$ coordinates are to be recorded and individual pixels identified. Signal from the inferred location is to be filtered through a band pass filter and amplified before connecting to a high speed analogue to digital converter (ADC).

\section{RESistive Charge Division Network}

Flat panel MAPMT H9500 from Hamamatsu photonics has been successfully applied in high resolution gamma imaging systems, such as positron emission tomography (PET) and single photon emission computed tomography (SPECT) scanners [14]. The MAPMT's high number of pixels offers exceptional angular resolution when coupled to appropriately selected sensitive detector. However, multiple anodes present a challenge associated with retrieving a high number of signals simultaneously. The challenge has been directly addressed by the development of readout electronics for H8500 and H9500 MAPMTs [11], [15] in gamma cameras.

Each of H9500 MAPMT's 256 anodes is a $2.8 \times 2.8 \mathrm{~mm}^{2}$ square, offering a high resolution results when incorporated in gamma-rays and neutron localisation systems [12], [15]. The sensitive area of $\mathrm{H} 9500$ is built of $16 \times 16$ matrix of individual pixels and is equal to $49 \times 49 \mathrm{~mm}^{2}$. If the signal appearing 
on the individual MAPMT's anode was to be processed independently, 256 ADC channels would be required to perform full analysis. The standard readout electronics circuit proposed by Popov and Majewski [15] reduces the number of required channels to 32 . Additional circuitry can also be added to the standard readout electronics to further reduce the number of the output channels to 4 ( 2 channels in $\mathrm{X}$ direction and 2 channels in $\mathrm{Y}$ direction). A schematic diagram of the standard readout electronics is presented in Fig. 1.

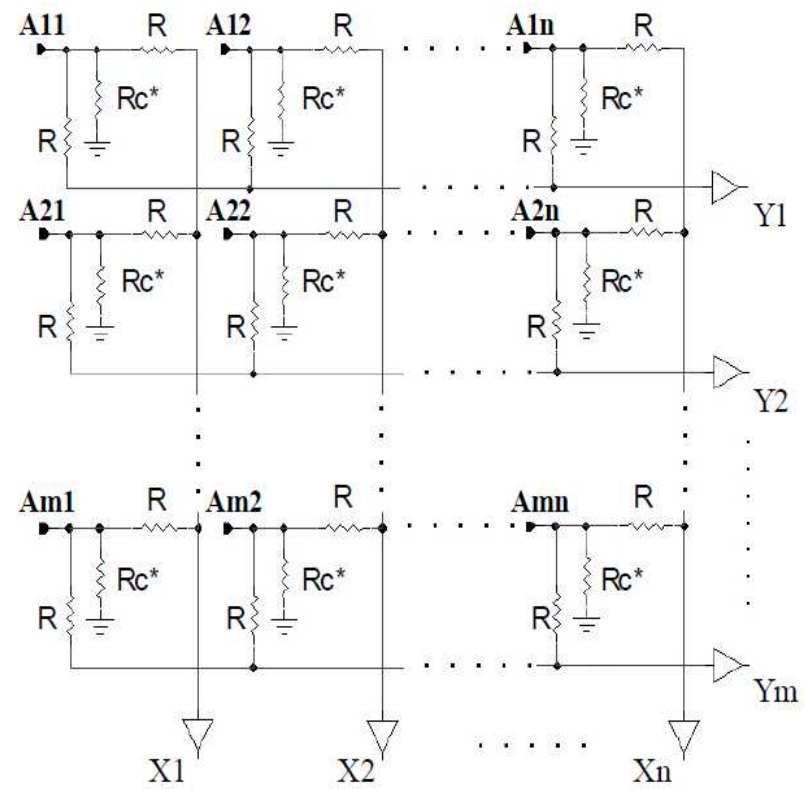

Fig. 1. Readout electronics circuit for 256 channels Hamamatsu H9500 MAPMT proposed by Popov and Majewski [15]

This method of positional data extraction has been successfully adapted in scintillator based high resolution gamma cameras. As crystal scintillators, such as $\mathrm{NaI}(\mathrm{Tl})$ and $\mathrm{CsI}(\mathrm{Tl})$, are sensitive exclusively to gamma-rays, number of interactions in a specific 2-D position can be counted, when light photons are detected on the anode of the MAPMT. Consequently, location of the gamma source can be detected with very high accuracy. It has been shown, that when tested with $\mathrm{NaI}(\mathrm{Tl})$ block scintillator, gamma ray interaction within an area of the single anode can be differentiated [15]. However, adaptation of the system for fast neutron imaging poses difficulties, as the scintillation detectors available are generally sensitive to both gamma-rays and neutrons.

Despite the difficulties, it was attempted to adopt the readout electronics circuit proposed by Popov and Majewski [15] for fast neutron radiography [12]. However, the system tested utilised EJ-200 plastic scintillator as a sensitive detector and was tested with D-D 2.5 MeV neutron generator. Although it showed accuracy of better than $0.5 \mathrm{~mm}$ in $\mathrm{X}$ and $\mathrm{Y}$ directions, its behaviour in mixed radiation environment cannot be assessed, as single radiation type environment was generated. Hence, further investigation and testing in mixed radiation fields would be required to fully evaluate the performance of the system.

In both cases (gamma and neutron detectors), the presented systems are only capable of detecting one type of radiation. Modern methods of fast neutron detection utilise PSD capable organic scintillators, which are sensitive to both gamma-ray and neutrons. A lack of detector sensitive exclusively to fast neutrons prevents the direct adaptation of the system from Fig. 1. for neutron localisation. Since organic plastic scintillator, such as EJ-299-33, detects fast neutrons as well as gamma-rays PSD would need to be performed on each individual channel to reliably class an event detected as neutron or gamma. For high resolution system based on one H9500, PSD would be required for all 256 channels.

\section{LTSPICE SIMULATION OF THE READOUT ELECTRONICS}

One way to reduce the number of channels would be to ensure that the signal extracted via readout electronics carries not only the positional data but also maintains the shape of the pulse, so that the origin of the interaction detected within the scintillator can be correctly identified. For testing purposes a schematic similar to the circuit shown in Fig. 1. was built in LTSpice simulation package for all 256 anodes of H9500 MAPMT. Simulated gamma and neutron pulses presented in Fig. 2. were connected to randomly chosen anodes in order to evaluate the signal appearing directly on 32 outputs.

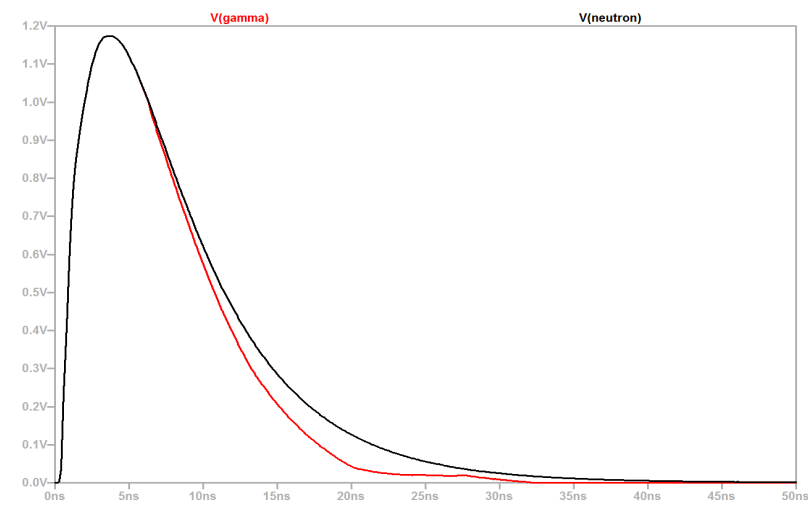

Fig. 2. Simulated gamma and neutron pulses connected to the resistive network

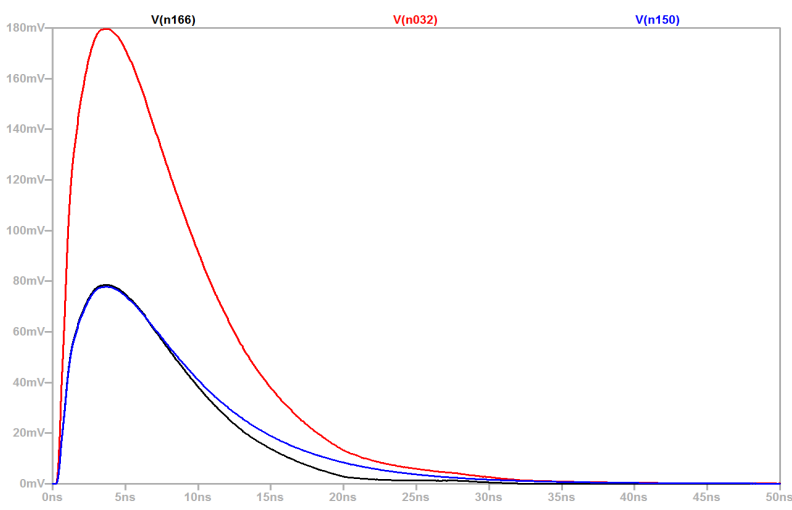

Fig. 3. Resistive network output signals probed at chosen locations

Simulated pulses were connected to two anodes and then probed at the two corresponding $\mathrm{X}$ outputs (black and blue 
traces) and single $\mathrm{Y}$ output (red trace) as reflected in Fig. 3. It shows that the pulses appear attenuated on the outputs. Amplification would be required before connecting to the $\mathrm{ADC}$, as lower resolution ADCs may not be capable of differentiating between very low sample values. Moreover, a way of separating pulses reaching multiple anodes simultaneously must also be addressed in the design of the filtering circuit. Finally, a development of an additional circuitry targeting noise, which appears within a resistive charge division readout methods [16], is required.

\section{Analogue to Digital Conversion for PSD}

Digital PSD has become a standard method of neutron identification in organic scintillation detectors, with Charge Comparison Method (CCM) algorithm being one of the most popular techniques implemented in the digital domain. It has proved superior to analogue zero crossing method [17]. On average, digital CCM performed $20 \%$ better than the zero crossing method implemented in the analogue domain.

The crucial part of a digital system implementing PSD is its ADC. Resolution and sampling frequency of an ADC determine the accuracy of the digitised signal, as well as discrimination precision between gamma-rays and neutron events. Generally, the higher the sampling rate the lower the resolution. Although the current state-of-the-art ADCs offer very high sampling rates, PSD has been successfully performed using 14-bit, 150 and 200 MSPS ADCs [18], [19]. Moreover, a recent research performed by Ruch et al. [20] suggest that 12-bit, 250 MSPS ADC provides sufficient accuracy for good PSD performance.

As a proof of concept, two Analog Devices AD9254 14bit, 150 MSPS ADCs on TERASIC AD/DA Data Conversion Card, were utilised. The data conversion card was connected to an Altera Cyclone IV EP4CE115 FPGA. As the data conversion card also contains a fast digital to analogue converter (DAC), 200 ns neutron and gamma pulses were generated for testing purposes. Pulses generated by the DAC were connected to the inputs of the two AD9254 ADCs. Outputs of the ADC channels were handled by a pair of ping-pong buffers implemented on the FPGA. Digitised samples were sent via $12 \mathrm{Mbits} / \mathrm{s}$ universal asynchronous receiver/transmitter (UART) to a computer running Linux. A Python script allowed for the implementation of high speed UART, resulting in a throughput of approximately 6000 samples/s.

Off-line analysis of the recorded samples was performed and reconstructed pulses plotted. These were analysed in order to assess their suitability for the digital CCM implementation. The digital CCM can be easily performed by the summation of all the ADC samples (long integral) and a number of samples associated with the tail of the pulse (short integral) [21]. Based on the ratio between the short and the long integral it can be determined if the pulse resulted from gamma or neutron interaction within the scintillator. The difference between the two generated pulses can be clearly recognised with the ADCs used. However, higher sampling rate ADC would be necessary for scintillation detectors producing faster light pulses than those generated in the simple test performed.

\section{CONCLUSION AND FUtURE PERSPECTIVE}

The work completed to date focused mainly on the development of FPGA code to extract sufficient number of ADC samples for digital PSD using CCM algorithm. Two ADCs present on the data conversion card allowed simultaneous processing of two channels. Although the shape of the reconstructed pulses suggests that higher sampling rate will be required, the outputs of two concurrently operating ADCs can be linked to the inputs of the rows and columns summing amplifiers to infer the location of the interaction. Furthermore, if the filtering circuit preserves sufficient information about the origin of the interaction in the scintillant, it should be possible to perform PSD for individual anode of the MAPMT.

Future work will be focused on the development of the filtering circuit for the positional PSD implementation. Also, emphasis will be placed on replacing AD9254 ADC with a 500 MSPS, 12-bit Texas Instruments ADS5463, which would enable input signal to be sampled every 2 ns. As it will have a significant impact on the accuracy of neutron/gamma discrimination, a faster FPGA may need to be introduced to provide reliable operation with the ADC of the higher sampling rate. When identified and localised, counted pulses will be combined with coded-aperture decoding algorithm to perform real-time source localisation. Once completed, the system is expected to combine high spatial resolution achieved in coded-aperture imaging systems with accurate neutron/gamma discrimination within organic plastic scintillator.

\section{ACKNOWLEDGEMENTS}

The authors would like to acknowledge the funding support from EPSRC via Faculty of Science and Technology, Lancaster University, UK and Sellafield Ltd., UK. We also acknowledge the help and advise of Mr Matthew Balmer at Lancaster University, UK.

\section{REFERENCES}

[1] C. Trotta, R. Massari, G. Trinci, N. Palermo, S. Boccalini, F. Scopinaro, and A. Soluri, "High-Resolution Imaging System (HiRIS) based on H9500 PSPMT," Nuclear Instruments and Methods in Physics Research, Section A: Accelerators, Spectrometers, Detectors and Associated Equipment, vol. 593, no. 3, pp. 454-458, 2008.

[2] P. Musico, E. Cisbani, F. Cusanno, F. Garibaldi, M. L. Magliozzi, S. Torrioli, G. Marano, M. Musumeci, M. Baiocchi, L. Vitelli, G. De Vincentis, S. Majewski, Tsui, and B, "A powerful readout system for high resolution and high efficiency molecular imaging studies of cardiovascular diseases in mice," pp. 1-3, 2009.

[3] L. Sinclair, P. Saull, D. Hanna, H. Seywerd, A. MacLeod, and P. Boyle, "Silicon photomultiplier-based compton telescope for safety and security (scotss)," IEEE Transactions on Nuclear Science, vol. 61, pp. 27452752, Oct 2014.

[4] S. Sun, Z. Zhang, L. Shuai, D. Li, Y. Wang, Y. Liu, X. Huang, H. Tang, T. Li, P. Chai, X. Jiang, B. Ma, M. Zhu, X. Wang, Y. Zhang, W. Zhou, F. Zeng, J. Guo, L. Sun, M. Yang, Y. Zhang, C. Wei, C. Ma, and L. Wei, "Development of a panorama coded-aperture gamma camera for radiation detection," Radiation Measurements, vol. 77, no. 0, pp. 34-40, 2015.

[5] S. Shifeng, Z. Zhiming, S. Lei, L. Daowu, W. Yingjie, L. Yantao, H. Xianchao, T. Haohui, L. Ting, C. Pei, Z. Yiwen, Z. Wei, Y. Mingjie, W. Cunfeng, M. Chuangxin, and W. Long, "Far field 3D localization of radioactive hot spots using a coded aperture camera," Applied Radiation and Isotopes, vol. 107, pp. 177-182, 2016. 
[6] E. Kowalski, Nuclear electronics [by] E. Kowalski. Springer-Verlag Berlin, New York, 1970.

[7] E. Brubaker and J. Steele, "Neutron imaging using the anisotropic response of crystalline organic scintillators," IEEE Nuclear Science Symposium Conference Record, pp. 1647-1652, 2010.

[8] Hamamatsu Photonics, Flat Panel Multianode Photomultiplier Tube, July 2011.

[9] Hamamatsu Photonics, Flat Panel Multianode Photomultiplier Tube, April 2015.

[10] J. T. Harris, P. M. Grudberg, and W. K. Warburton, "Digital electronics for 256 anode Hamamatsu H9500 PSPMT arrays in full-volume Compton imagers," Journal of Instrumentation, vol. 9, no. 07, pp. C07005C07005, 2014.

[11] P. D. Olcott, J. A. Talcott, C. S. Levin, F. Habte, and A. M. K. Foudray, "Compact readout electronics for position sensitive photomultiplier tubes," IEEE Transactions on Nuclear Science, vol. 52, no. 1 I, pp. $21-$ 27, 2005.

[12] V. Popov, P. Degtiarenko, and I. Musatov, "New detector for use in fast neutron radiography," Journal of Instrumentation, vol. 6, no. 01, pp. C01029-C01029, 2011.

[13] V. Popov, "Advanced data readout technique for Multianode Position Sensitive Photomultiplier Tube applicable in radiation imaging detectors," Journal of Instrumentation, vol. 6, no. 01, pp. C01061-C01061, 2011.

[14] J. Proffitt, W. Hammond, S. Majewski, V. Popov, R. R. Raylman, and A. G. Weisenberger, "Implementation of a high-rate USB data acquisition system for PET and SPECT imaging," IEEE Nuclear Science Symposium Conference Record, vol. 5, pp. 3063-3067, 2007.

[15] V. Popov and S. Majewski, "A compact high performance readout electronics solution for H9500 Hamamatsu 256 Multianode Photomultiplier tube for application in gamma cameras," IEEE Nuclear Science Symposium Conference Record, vol. 5, pp. 2981-2985, 2007.

[16] J. C. Vermeulen, "Noise in resistive charge-division position-sensing methods," Nuclear Instruments and Methods, vol. 185, no. 1-3, pp. 591593, 1981.

[17] C. Sosa, M. Flaska, and S. Pozzi, "Comparison of analog and digital pulse-shape-discrimination systems," Nuclear Instruments and Methods in Physics Research Section A: Accelerators, Spectrometers, Detectors and Associated Equipment, vol. 826, pp. 72-79, 2016.

[18] M. Balmer, K. Gamage, and G. Taylor, "Neutron assay in mixed radiation fields with a ${ }^{6} \mathrm{Li}$-loaded plastic scintillator," Journal of Instrumentation, vol. 10, no. 08, pp. P08012-P08012, 2015.

[19] N. Zaitseva, A. Glenn, H. Paul Martinez, L. Carman, I. Pawe??czak, M. Faust, and S. Payne, "Pulse shape discrimination with lithiumcontaining organic scintillators," Nuclear Instruments and Methods in Physics Research, Section A: Accelerators, Spectrometers, Detectors and Associated Equipment, vol. 729, pp. 747-754, 2013.

[20] M. L. Ruch, M. Flaska, and S. A. Pozzi, "Pulse shape discrimination performance of stilbene coupled to low-noise silicon photomultipliers," Nuclear Instruments and Methods in Physics Research, Section A: Accelerators, Spectrometers, Detectors and Associated Equipment, vol. 793, pp. 1-5, 2015.

[21] K. Gamage, M. Joyce, and N. Hawkes, "A comparison of four different digital algorithms for pulse-shape discrimination in fast scintillators," Nuclear Instruments and Methods in Physics Research Section A: Accelerators, Spectrometers, Detectors and Associated Equipment, vol. 642, no. 1 , pp. 78-83, 2011. 